\title{
Designed Oil Products from Cracked Bottom Oil*
}

\author{
by Shimpei Gomi** and Masaaki Takahashi**
}

\begin{abstract}
Summary: The process of utilizing the cracked bottom oil and the new oil products called "designed oils" has been developed and the first semicommercial plant is now under successful operation.

The process consists of three steps namely hydrotreating, alkylation or hydrogenation, and fractionation. Special catalysts have been developed for treating heavy cracked bottom oils and two types of oils having unique cyclic structure are obtained by this process.

Many interesting applications such as heat transfer oils, electrical insulating oils, epoxy and urethane diluents and special solvents have been found and are now being markeled.

This paper describes the process of utilizing cracked bottom oil and characteristics of various new oil products.
\end{abstract}

\section{Introduction}

The cracked bottom oil obtained by thermal cracking process for production of olefins has never been considered seriously as a new source of valuable chemical products. In this cracking process, light hydrocarbons such as naphtha are usually used as a feed stock. Recently however, heavier hydrocarbons such as kerosene, gas oil or even crude oil have been considered as more favorable feed stock for olefin production.

Kureha Chemical Industry Co., Ltd. developed the high temperature crude oil cracking process and the first commercial plant is now under successful operation. This crude oil cracking is carried out under conditions of higher temperature and shorter residence time than conventional naphtha cracking condition, using high temperature steam $\left(2,000^{\circ} \mathrm{C}\right)$ as heat carrier, and comparatively large amounts of cracked bottom oil are obtained. It is of utmost importance that the bottom oil should be used as a new source of valuable chemical products, in order to achieve better over all economics.

We have developed a process of utilizing the bottom oil, and many new products have been commercialized through this process. The new products can be called "designed oils" by their characteristics and the way of applications. The first semi-commercial plant (capacity of 15,000 tons per year) producing designed oils from cracked bottom which is obtained by high tem-

* Received December 7, 1970.

** Kureha Chemical Industry Co., Ltd. (1-8, Nihonbashi, Horidome-cho, Chuo-ku, Tokyo, Japan)

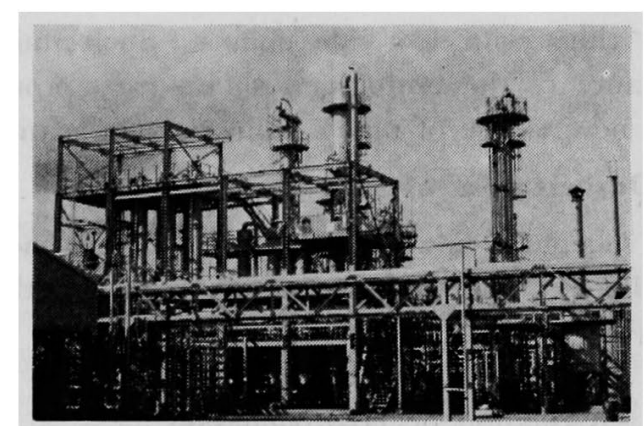

Fig. 1 Semi-Commercial Plant for Designed Oil Production

perature crude oil cracking was put on stream by Kureha C'hemical Industry Company in October, 1970.

\section{Properties of Cracked Bottom Oil}

The remarkable characteristic of cracked bottom oil is that since it is produced at higher temperature, complete aromaticity is guaranteed for this fraction. In other words, structural rearrangement of feed stock can be carried out at high temperature and aromatic hydrocarbon becomes the only liquid product throughout the process. The bottom oil contains mainly aromatic hydrocarbon having two or more ben-

Table 1 Typical Properties of Cracked Bottom Oil

\begin{tabular}{ll|l}
\hline Density $d^{15}$ & & 1.025 \\
Refractive Index & $n_{\mathrm{D}}^{25}$ & 1.615 \\
Sulfur, \% & & 0.1 \\
Bromine No. & & 3 \\
Diene Value & 5 \\
Distillation & IBP & $163^{\circ} \mathrm{C}$ \\
& $10 \%$ & 203 \\
& $30 \%$ & 215 \\
& $50 \%$ & 232 \\
& $70 \%$ & 275 \\
& $90 \%$ & 350 \\
\hline
\end{tabular}




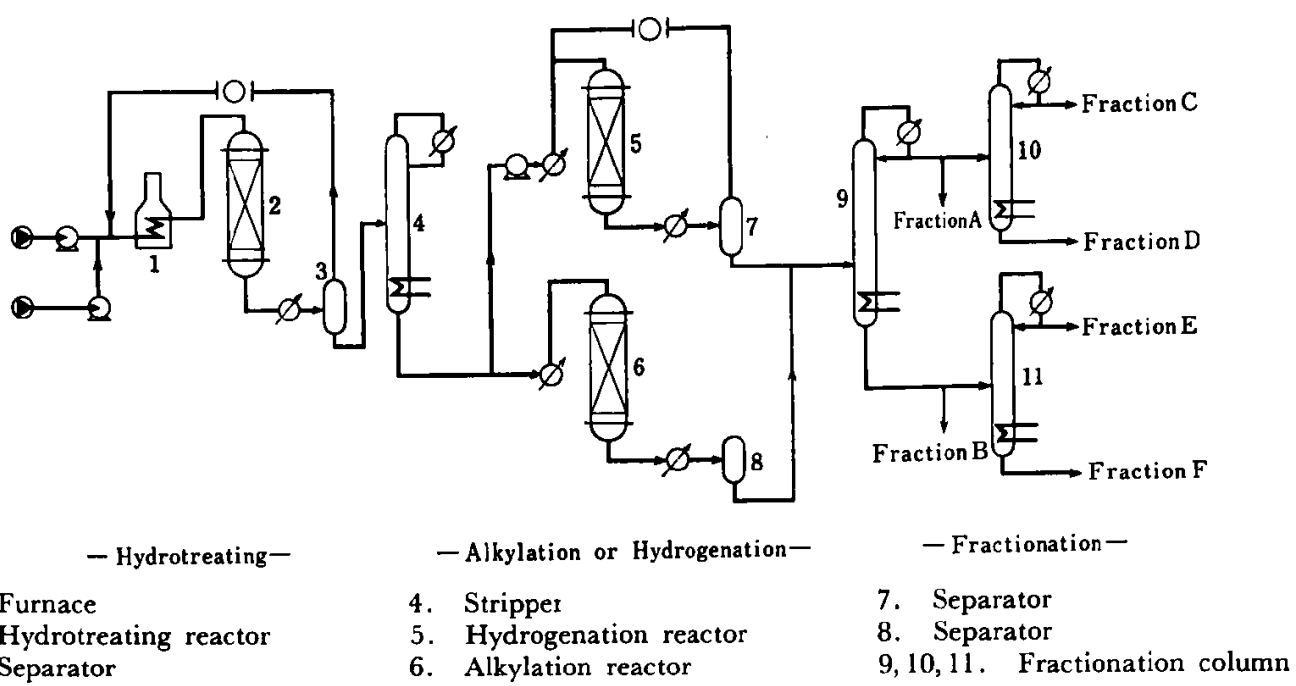

Fig. 2 Schematic Flow Sheet of the Process

zene rings with less side chains. Such unique structure of the bottom oil shows great promise as a new source of many industrial applications.

\section{Description of the Process}

A schematic flow sheet of the process is shown in Fig. 2. The process contains essestially three steps:

1. Hydrotreating

2. Alkylation or Hydrogenation

3. Distillation

Hydrotreating is carried out in the presence of solid catalyst and hydrogen under pressure. Most of sulfur and basic nitrogen are removed and saturation of unstable olefins also occurs in this step. The reaction condition of hydrotreating can be varied depending on the nature of feed stocks. Usually the pressure and temperature range are $15 \sim 45 \mathrm{~kg} / \mathrm{cm}^{2} \mathrm{G}$ and $300 \sim 400^{\circ} \mathrm{C}$, respectively. In alkylation reaction, special solid catalyst is used and reaction is carried out at moderate pressure in the continuous flow system.

The lighter fraction of cracked bottom oil (B.P. range ca. $200 \sim 300^{\circ} \mathrm{C}$ ) is usually alkylated with ethylene, propylene or higher olefins. Through this alkylation step, various types of alkylpolyaromatic hydrocarbons are obtained. On the other hand, the heavier fraction of cracked bottom oil (B.P. range ca. $300 \sim 450^{\circ} \mathrm{C}$ ) is usually subject to complete hydrogenation. This step is carried out at high pressure (above $100 \mathrm{~kg}$ / $\mathrm{cm}^{2} \mathrm{G}$ ) but at moderate reaction temperature (below $300^{\circ} \mathrm{C}$ ) by using fixed bed hydrogenation catalyst. Polycycloparaffinic hydrocarbons are the reaction products of this step. Thus, two types of oils are obtained by the second step. These two types-alkylpolyaromatics and polycycloparaffinics- are rather new in the industrial sense. The final step is distillation where these two types of oils are fractionated in order to match the end use. In case blending of the different types of oils is necessary, this can be done in the line blending system before shipping. In terms of boiling point, these oil products are approximately the same level as conventional lubricating oil fractions from petroleum refinery. But in the structural sense, these oils are completely different from the lube oil. Lube oil is normally separated from crude oil and purified by several techniques such as solvent extraction, acid treating, or hydrotreating, and it is a complicated mixture of $n$-paraffins, isoparaffins, naphthenes, and aromatics. However, both new oil products are pure homologues of either alkylaromatics or polycycloparaffinics (complete naphthenes) in their structure.

The structure of alkylaromatic oil is mainly two-ring aromatics (e.g. naphthalene, biphenyl, acenaphthene, fluorene) having $\mathrm{C}_{2} \sim \mathrm{C}_{4}$ alkyl side chains. On the other hand, the polycycloparaffinic oil is mainly three to five-ring, completely saturated naphthenes.

Many new applications are based upon this unique structure and the main advantage is that one can "design" the oil products in terms of structure and properties for the customer in order to make "the best fit" oil products.

\section{Oil Products}

Since these two types of oils have very unique structure, their properties are also expected to 
be interesting. The basic properties which were found until now are summarized as follows;

a. high thermal stability

b. low pour points

c. high solvency for polymers and dyes

d. good electrical properties

e. low toxicity

\subsection{Heat Transfer Oil}

Because of excellent thermal stability, several

Table 2 Properties KSK Oils

\begin{tabular}{|c|c|c|c|c|}
\hline \multicolumn{2}{|c|}{ Commercial Name } & KSK -260 & KSK-280 & KSK -330 \\
\hline \multirow{2}{*}{\multicolumn{2}{|c|}{$\begin{array}{l}\text { Color, Union } \\
\text { Molecular Weight } \\
\text { Density }\left(20^{\circ} \mathrm{C}\right) \\
\text { Boiling Point }\left({ }^{\circ} \mathrm{C}\right) \\
\text { Pour Point }\left({ }^{\circ} \mathrm{C}\right) \\
\text { Specific Heat (at b. p.) } \\
\text { Latent Heat of Evapora- } \\
\text { tion (kcal } / \mathrm{kg}) \\
\text { Volume Expansion } \\
\text { Coefficient } \\
\text { Flash Point (P.M.) ('C) }\end{array}$}} & \begin{tabular}{|c}
$<1$ \\
180 \\
0.96 \\
268 \\
$<-50$ \\
0.60 \\
64 \\
$7.9 \times 10^{-}$
\end{tabular} & $\begin{array}{c}<1 \\
210 \\
0.96 \\
295 \\
<-40 \\
0.62 \\
59\end{array}$ & $\begin{array}{l}<1 \\
250 \\
0.96 \\
323 \\
<-30 \\
0.65 \\
51\end{array}$ \\
\hline & & 120 & 145 & 165 \\
\hline $\begin{array}{l}\text { Viscosity } \\
\text { (cP) }\end{array}$ & $\begin{array}{r}-10^{\circ} \mathbf{C} \\
0^{\circ} \mathrm{C} \\
5^{\circ} \mathrm{C} \\
10^{\circ} \mathrm{C} \\
20^{\circ} \mathrm{C} \\
100^{\circ} \mathrm{C} \\
200^{\circ} \mathrm{C} \\
300^{\circ} \mathrm{C} \\
340^{\circ} \mathrm{C}\end{array}$ & $\begin{array}{r}19.7 \\
12.0 \\
10.8 \\
8.2 \\
5.8 \\
1.0 \\
0.30 \\
0.15 \\
0.13\end{array}$ & $\begin{array}{l}89.5 \\
41.2 \\
32.0 \\
24.5 \\
15.5 \\
1.4 \\
0.35 \\
0.17 \\
0.14\end{array}$ & $\begin{array}{c}830.0 \\
231.0 \\
182.0 \\
121.5 \\
55.0 \\
3.2 \\
0.66 \\
0.27 \\
0.21\end{array}$ \\
\hline $\begin{array}{l}\text { Thermal Conduc- } \\
\text { tivity (Liquid) } \\
\mathrm{kcal} / \mathrm{m} \cdot \mathrm{hr}{ }^{\circ} \mathbf{C}\end{array}$ & $\begin{array}{l}100^{\circ} \mathrm{C} \\
200^{\circ} \mathrm{C} \\
300^{\circ} \mathrm{C} \\
340^{\circ} \mathrm{C}\end{array}$ & $\begin{array}{l}0.100 \\
0.094 \\
0.088 \\
0.085\end{array}$ & $\begin{array}{l}0.105 \\
0.094 \\
0.088 \\
0.085\end{array}$ & $\begin{array}{l}0.101 \\
0.095 \\
0.089 \\
0.087\end{array}$ \\
\hline $\begin{array}{l}\text { Vapour Pressure } \\
\mathrm{kg} / \mathrm{cm}^{2} \mathbf{G}\end{array}$ & $\begin{array}{l}220^{\circ} \mathrm{C} \\
240^{\circ} \mathrm{C} \\
260^{\circ} \mathrm{C} \\
280^{\circ} \mathrm{C} \\
300^{\circ} \mathrm{C} \\
320^{\circ} \mathrm{C} \\
340^{\circ} \mathrm{C}\end{array}$ & $\begin{array}{r}-0.60 \\
-0.42 \\
-0.15 \\
0.22 \\
0.80 \\
1.65 \\
2.85\end{array}$ & $\begin{array}{r}-0.70 \\
-0.65 \\
-0.50 \\
-0.25 \\
0.10 \\
0.60 \\
1.30\end{array}$ & $\begin{array}{r}-0.50 \\
-0.30 \\
-0.02 \\
0.45\end{array}$ \\
\hline
\end{tabular}

fractions of alkyl aromatics can be used as good heat transfer oils. Three types of heat transfer oils are available commercially though Soken Chemical Engineering Company and Kureha Chemical Industry Company under the name of KSK-260, 280 and 330 (Table 2).

Characteristics of KSK Oils

1. Excellent thermal stability, particularly low sludge formation.

2. Very low pour point

3. Non-corrosive

4. Low toxicity and odorless.

\section{Application}

1. Industrial heating system reactor, heat exchanger, distillation

2. Plastics or fiber fabrication

3. Snow melting system

Bus stops, high way, shopping area, etc.

4. Panel heating system in housing

5. Cooling system

\subsection{Electrical Insulating Oil}

Excellent electrical insulating oils are obtained from both types of oils based on good electrical properties together with good thermal stability.

The lower fraction of alkylaromatic oil can be used as ultra high voltage cable oils having excellent dielectric properties. Table 3 shows these characteristics in comparison with dodecylbenzene which is now used as high voltage cable oil.

By blending the two types of oil, one can obtain a very interesting insulating oil which is used for new type of transformer (Fig. 3).

This new application is based on the fact that

Table 3 Properties of Cable Oil

\begin{tabular}{|c|c|c|c|}
\hline & Cable Oil-A* & Cable Oil-B** & Dodecylbenzene \\
\hline $\begin{array}{l}\text { Color, Union } \\
\text { Density } d^{15}{ }^{4} \mathrm{CSt} 100^{\circ} \mathrm{F} \\
\text { Viscosity, cSt } 210^{\circ} \mathrm{F} \\
\text { Pour Point, }{ }^{\circ} \mathrm{C} \\
\text { Flash Point, }{ }^{\circ} \mathrm{C} \\
\text { Evaporation Loss, } \%\left(98^{\circ} \mathrm{C}, 5 \mathrm{hrs}\right) \\
\text { Acid Value, } \mathrm{mg} \mathrm{KOH} / \mathrm{g} \\
\text { Copper Corrosion Test, } 100-\mathrm{C} \times 3 \mathrm{hrs} \\
\text { Dielectric Properties }\left(80^{\circ} \mathrm{C}\right) \\
\quad \varepsilon \\
\text { tan } \delta, \% \\
\rho, \Omega \text {-cm } \\
\text { Aging Test (Open beaker, } 100^{\circ} \mathrm{C} \times 96 \mathrm{hrs} \\
\text { with copper) } \\
\quad \text { tan } \delta, \% \\
\rho \\
\text { Break-down Voltage, kV } / \mathrm{mm} \\
\text { (Oil impregnated paper) } \\
\text { A. C. } \\
\text { Impulse }\end{array}$ & $\begin{array}{c}<1 \text { (colorless) } \\
0.953 \\
7.4 \\
1.7 \\
<-50 \\
142 \\
\quad 0.18 \\
0.004 \\
\text { la } \\
2.42 \\
0.006 \\
1.5 \times 10^{16} \\
\\
0.101 \\
1.6 \times 10^{14}\end{array}$ & $\begin{array}{l}<1 \text { (colorless) } \\
0.965 \\
9.1 \\
2.4 \\
<-50 \\
135 \\
\quad 0.10 \\
0.003 \\
1 \mathrm{a} \\
\\
2.24 \\
0.001 \\
4.0 \times 10^{16}\end{array}$ & $\begin{array}{c}<1 \text { (colorless) } \\
0.868 \\
7.6 \\
1.9 \\
<-50 \\
136 \\
0.21 \\
0.003 \\
1 \mathrm{a} \\
\\
2.19 \\
\mathbf{0 . 0 0 4} \\
5.0 \times 10^{16}\end{array}$ \\
\hline
\end{tabular}

* Alkylpolyaromatic oil

** Polycycloparaffinic oil

Volume 13, No. 1, May 1971 


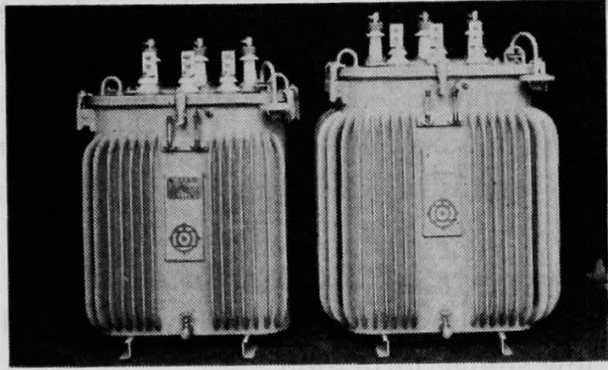

Fig. 3. Left: New Type Transformer (B-Class) using New Oil

Right: Conventional Transformer (A-Class) using Mineral Oil

Table 4 Properties of New Transformer Oil

\begin{tabular}{|c|c|c|}
\hline & $\begin{array}{l}\text { New Trans- } \\
\text { former Oil }\end{array}$ & $\begin{array}{c}\text { Mineral Oil } \\
\text { (JIS } \sharp 2)\end{array}$ \\
\hline Specific Gravity, $d^{15}$ & 0.97 & 0.87 \\
\hline $\begin{array}{c}\text { Kinematic Viscosiy, } \\
100^{\circ} \mathrm{F} \\
210^{\circ} \mathrm{F}\end{array}$ & $\begin{array}{r}21.6 \\
3.2\end{array}$ & $\begin{array}{r}11.0 \\
2.6\end{array}$ \\
\hline $\begin{array}{l}\text { Flash Point (P. M.), }{ }^{\circ} \mathrm{C} \\
\text { Pour Point, }{ }^{\circ} \mathrm{C}\end{array}$ & $\begin{array}{l}160 \\
-35\end{array}$ & $\begin{array}{l}135 \\
-45\end{array}$ \\
\hline $\begin{array}{l}\text { Evaporation Loss } \\
\left(98^{\circ} \mathrm{C}, 5 \mathrm{hrs}\right), \%\end{array}$ & 0.1 & 0.2 \\
\hline $\begin{array}{l}\text { Oxidation Stability (JIS } \\
\mathrm{C}-2101,120^{\circ} \mathrm{C}, 75 \mathrm{hrs}, \\
\text { with } \mathrm{O}_{2} \text { and } \mathrm{Cu} \text { wire) }\end{array}$ & & \\
\hline $\begin{array}{l}\text { Acid Value mg KOH/g } \\
\text { Sludge, \% } \\
\text { Dielectric Constant }\end{array}$ & $\begin{array}{l}0.11 \\
0.02\end{array}$ & $\begin{array}{l}0.21 \\
0.12\end{array}$ \\
\hline $\begin{array}{cr}\text { Dielectric Constant } \\
& 80^{\circ} \mathrm{C} \\
\varepsilon & 100^{\circ} \mathrm{C} \\
120^{\circ} \mathrm{C}\end{array}$ & $\begin{array}{l}2.45 \\
2.40 \\
2.30\end{array}$ & $\begin{array}{l}2.17 \\
2.14 \\
2.11\end{array}$ \\
\hline Dielectric Loss & & \\
\hline $\begin{array}{ll} & 80^{\circ} \mathrm{C} \\
\tan \delta(\%) & 100^{\circ} \mathrm{C} \\
120^{\circ} \mathrm{C}\end{array}$ & $\begin{array}{l}0.024 \\
0.052 \\
0.080\end{array}$ & $\begin{array}{l}0.074 \\
0.096 \\
0.239\end{array}$ \\
\hline 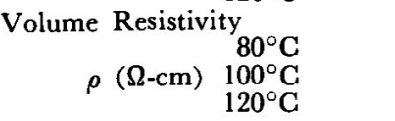 & $\begin{array}{l}9.5 \times 10^{15} \\
5.1 \times 10^{15} \\
1.7 \times 10^{15}\end{array}$ & $\begin{array}{l}3.9 \times 10^{14} \\
1.2 \times 10^{14} \\
8.6 \times 10^{13}\end{array}$ \\
\hline
\end{tabular}

degradation of insulating paper is greatly depressed by using the new oil in the transformer system, so that reduction of transformer size and cost can be accomplished by using this new insulation system which can operate at higher temperatures.

The physical and electrical properties of the new transformer oils are shown in Table 4. Fig. 4 and 5 shows the aging of insulating paper (craft and cyanoethylated paper) in an model transformer.

The transformer which has B-class thermal stability $\left(130^{\circ} \mathrm{C}\right.$ of coil temperature) has been manufactured and is now under operation.

\subsection{Epoxy and Urethane Diluent}

Heavier fractions of alkylated polyaromatic oil serve as diluent and modifier for epoxy and urethane resins without loss of its basic characteristics, and have wide applications with many advantages.

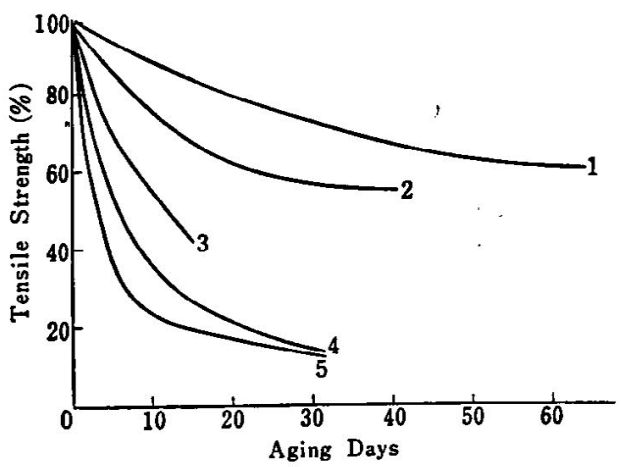

Comparison of tensile strength of Kraft Paper with new transformer oil and conventional transformer oil

$\begin{array}{llcc}\text { 1. New transformer oil } & \text { Aging temp. } 120^{\circ} \mathrm{C} \\ \text { 2. } & \text { " } & 130 \\ \text { 3. " } & 150 \\ \text { 4. Conventional transformer oil " } & 120 \\ \text { 5. " } & & 130 \\ \text { Fig. } 4 & \text { Aging Test at Various Temperature }\end{array}$

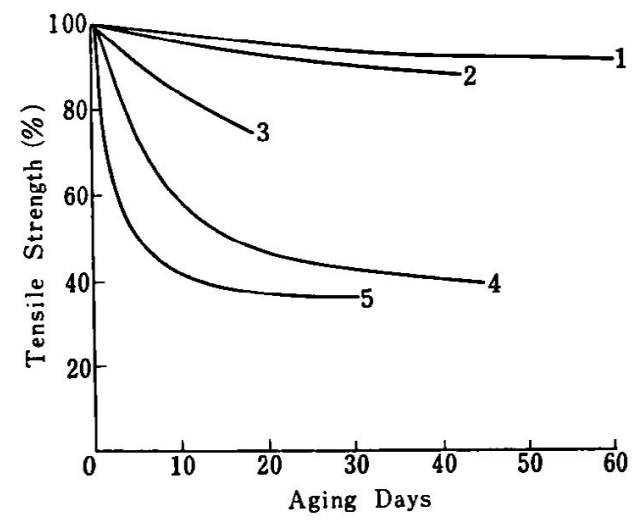

Comparison of tensile strength of Cyano Kraft with new transformer oil and conventional transformer oil

$\begin{array}{lcc}\text { 1. New transformer oil } & \text { Aging temp. } 120^{\circ} \mathrm{C} \\ \text { 2. " } & \text { " } & 130 \\ \text { 3. } & \text { " } & 150 \\ \text { 4. Conventional transformer oil " } & 120 \\ \text { 5. " } & \text { " } & 130\end{array}$

Fig. 5 Aging Test at Various Temperature

Typical characteristics are,

1. good compatibility and workability

2. improve chemical and water resistance

3. good electrical properties

4. low volatility

5. storage stability

Table 5 shows general properties of such diluents, and the properties of cured products are shown in Table 6 and Fig. 6, 7.

\subsection{Special Solvents}

A variety of solvents having different boiling ranges can be obtained by distillation of either hydrogenated or alkylated polyaromatic hydrocarbons.

The solvents have many interesting appli- 
cations based on their characteristics such as low volatility, low pour point, and good solvency for polymers and dyes. Table 7 shows the properties of the solvents.

Table 5 General Properties

\begin{tabular}{l|c|c|c}
\hline $\begin{array}{c}\text { Name of } \\
\text { Diluent }\end{array}$ & EX-50 & EX-1500 & UZ-500 \\
\hline $\begin{array}{c}\text { Use } \\
\text { Viscosity, } \\
\text { cP }\left(25^{\circ} \mathrm{C}\right)\end{array}$ & $\begin{array}{c}\text { Low viscosity } \\
\text { epoxy diluent }\end{array}$ & $\begin{array}{c}\text { High viscosity } \\
\text { epoxy diluent }\end{array}$ & $\begin{array}{c}\text { Urethane } \\
\text { diluent }\end{array}$ \\
$\begin{array}{c}\text { Density, 25 } \\
\text { Evaporation } \\
\text { Loss, } \%\end{array}$ & 0.98 & $1,500 \sim 2,000$ & $600 \sim 800$ \\
$110^{\circ} \mathbf{C} 3$ hrs & $<1$ & $<00$ & 1.00 \\
Color & Colorless & $\begin{array}{c}\text { Gardner No. } \\
9 \sim 11\end{array}$ & $\begin{array}{c}\text { Gardner No. } \\
6 \sim 8\end{array}$ \\
\hline
\end{tabular}
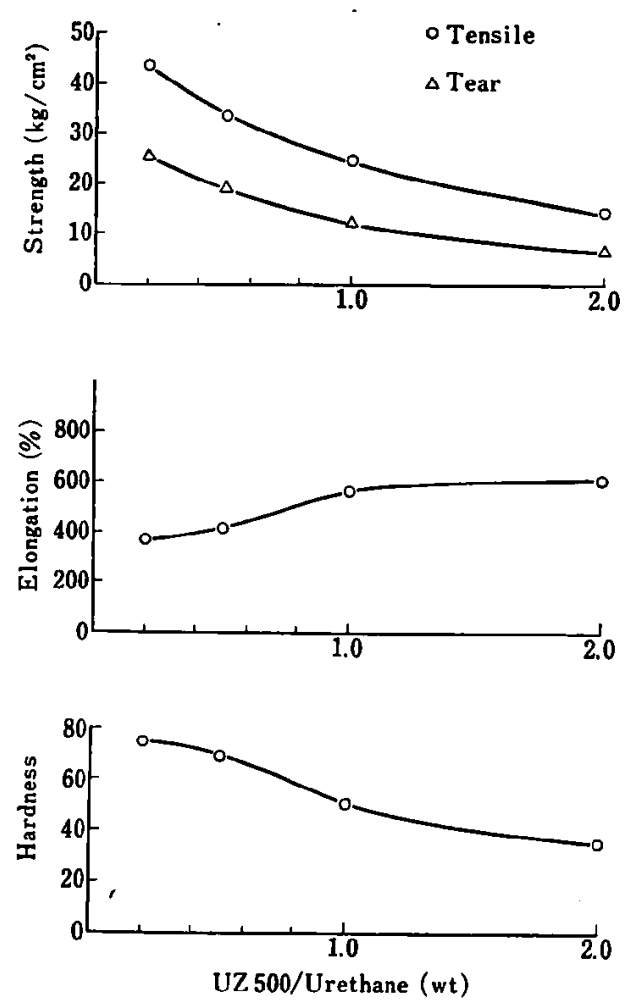

Fig. 6 Mechanical Properties of UZ 500 Polyurethane (Sealant Type, MOCA Curing)
The solvents can be useful in painting, printing inks, polymer processing or insecticide formulation.

Very interesting and promising application has been found recently which is the solvent of

Table 6 Properties of Cured Products of Epoxy-EX 1500 System

\begin{tabular}{|c|c|c|c|}
\hline & $\underset{A}{\text { Sample }}$ & $\underset{\mathrm{B}}{\text { Sample }}$ & $\underset{\mathrm{C}}{\text { Sample }}$ \\
\hline $\begin{array}{l}\text { Recipe: } \\
\text { Epikote } 815 \\
\text { EX-1500 } \\
\text { Triethylenetetramine } \\
\text { Phenol }\end{array}$ & $\mid \begin{array}{c}\text { PHR } \\
100 \\
\overline{12} \\
3\end{array}$ & \begin{tabular}{|c|} 
PHR \\
100 \\
50 \\
12 \\
3
\end{tabular} & $\begin{array}{l}\text { PHR } \\
100 \\
100 \\
12 \\
3\end{array}$ \\
\hline Curing Condition & $\mid \begin{array}{c}20^{\circ} \mathrm{G} \\
7 \text { days }\end{array}$ & $\mid \begin{array}{c}20^{\circ} \mathrm{C} \\
7 \text { days }\end{array}$ & $\begin{array}{l}20^{\circ} \mathrm{C} \\
7 \text { days }\end{array}$ \\
\hline $\begin{array}{l}\text { Adhesive Strength, } \mathrm{kg} / \mathrm{cm}^{2} \\
\text { (ASTM D 1002) }\end{array}$ & 50 & 87 & 70 \\
\hline $\begin{array}{l}\text { Hardness (Rockwell, M) } \\
\text { Impact Strength } \mathrm{kg}-\mathrm{cm} / \mathrm{cm}^{2} \\
\text { (Charpy, with notch) }\end{array}$ & $\begin{array}{r}100 \\
2.0\end{array}$ & $\begin{array}{l}82 \\
2.0\end{array}$ & $\begin{array}{l}73 \\
2.0\end{array}$ \\
\hline $\begin{array}{l}\text { Tensile Strength, } \mathrm{kg} / \mathrm{mm}^{2} \\
\text { Elongation, \% }\end{array}$ & $\begin{array}{l}3.4 \\
8.0\end{array}$ & $\begin{array}{l}3.2 \\
8.0\end{array}$ & $\begin{array}{l}3.0 \\
8.2\end{array}$ \\
\hline
\end{tabular}
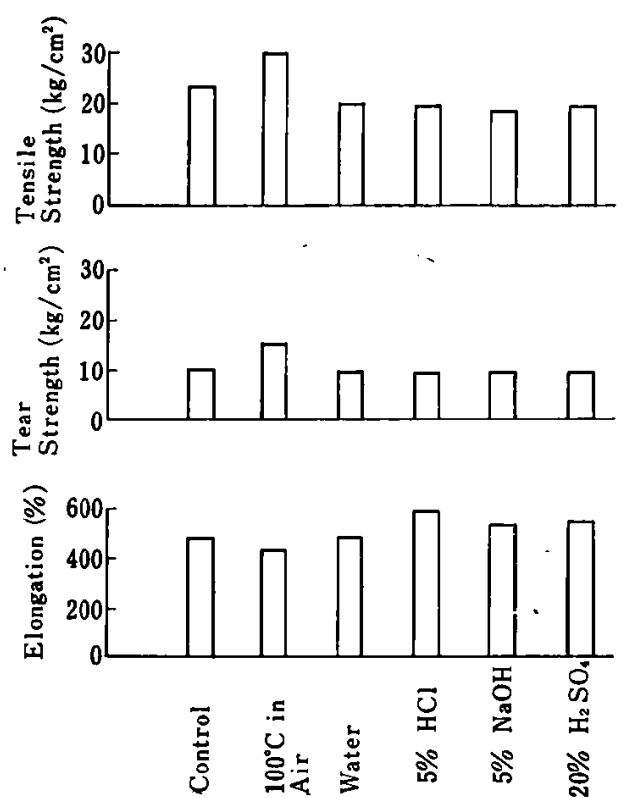

Fig. 7 Heat, Water and Chemical Resistance of $\mathrm{UZ}$ 500 Modified Polyurethane After One Week (UZ 500/Prepolymer =1/1, MOCA Curing)

Table 7 Properties of Special Solvents

\begin{tabular}{|c|c|c|c|c|}
\hline Solvents & A & B & C & $\mathrm{D}$ \\
\hline $\begin{array}{l}\text { Type of Hydrocarbon } \\
\text { Average Boiling Point, }{ }^{-} \mathrm{C} \\
\text { Refractive Index } n_{\mathrm{D}}^{25} \\
\text { Flash Point, (P.M.) }{ }^{\circ} \mathrm{C} \\
\text { Viscosity, cSt }\end{array}$ & $\begin{array}{c}\text { Polycycloparaffin } \\
300 \\
1.514 \\
135\end{array}$ & $\begin{array}{c}\text { Polycycloparaffin } \\
330 \\
1.536 \\
160\end{array}$ & $\begin{array}{c}\text { Alkylpolyaromatics } \\
270 \\
1.550 \\
115\end{array}$ & $\begin{array}{c}\text { Alkylpolyaromatics } \\
300 \\
1.560 \\
140\end{array}$ \\
\hline $\begin{aligned} & 30^{\circ} \mathrm{C} \\
& 75^{\circ} \mathrm{C}\end{aligned}$ & $\begin{array}{r}12.0 \\
3.7\end{array}$ & $\begin{array}{r}38.2 \\
6.6\end{array}$ & $\begin{array}{l}3.7 \\
1.5\end{array}$ & $\begin{array}{r}10.4 \\
2.7\end{array}$ \\
\hline $\begin{array}{l}\text { Evaporation Loss, } \% \\
\quad\left(100^{\circ} \mathrm{C}, 5 \mathrm{hr}\right) \\
\text { Vapor Pressure, } \mathrm{mmHg}\end{array}$ & $<0.1$ & $<0.05$ & $<1.5$ & $<0.2$ \\
\hline $\begin{array}{r}100^{\circ} \mathrm{C} \\
200^{\circ} \mathrm{C} \\
\text { Kauri Butanol Number }\end{array}$ & $\begin{array}{r}1 \\
63 \\
120\end{array}$ & $\begin{array}{l}0.5 \\
25 \\
65\end{array}$ & $\begin{array}{l}3.4 \\
160 \\
70\end{array}$ & $\begin{array}{r}1 \\
72 \\
55\end{array}$ \\
\hline
\end{tabular}

Volume 13, No. 1, May 1971 
dye in micro-capsule for carbonless paper system.

\section{Conclusion}

Single ring aromatics such as benzene, toluene xylenes and their derivatives are commercially available in large volume and have been found many applications mainly in the field of plastics, synthetic fibers, or synthetic rubbers.

However, only a few polyaromatic hydrocarbons have been utilized commercially. Phthalic anhydride from naphthalene is the only large volume application, but even in this case, naphthalene is gradually being replaced by $o$-xylene.

The new oil products from mixed polyaromatic hydrocarbons are now finding many interesting applications as a new type of valuable chemical product. instead of being used as fuel or cheaper raw materials like carbon black oil.

With continuous effort for developing new areas of polyaromatic hydrocarbon chemistry, the authors believe that there will be many fruitful possibilities of creating new products from these unique polyaromatic streams.

\section{Acknowledgement}

The authors gratefully acknowledge the contributions and cooperation of all, particularly Japan Gasoline Company, who participated in the development of the new process and related products described herein.

The authors would also like to express their special appreciation to Mr. Saburo Araki, President of Kureha Chemical Industry Co., Ltd. for his consistent encouragement to the development. 